\title{
A Comparison between Drilling and Standard Penetration Test (SPT) Data to the Electrical Resistivity Sounding with Schlumberger Configuration in UNS Area
}

\author{
Sinta Nur Rizqi Listanti ${ }^{1}$, Darsono Darsono ${ }^{1^{*}}$, and Yusep Muslih Purwana ${ }^{2}$ \\ ${ }^{1}$ Physics Department, Faculty Mathematics and Natural Science, Universitas Sebelas Maret Surakarta \\ ${ }^{2}$ Civil Engineering, Faculty of Engineering, Universitas Sebelas Maret Surakarta \\ "Email: onos.dar2014@gmail.com
}

\begin{abstract}
The geophysics test using electrical resistivity method has been conducted in the area of UNS Campus to indicate the lithological of subsurface. This method is categorized as a Non-Destructive Test (NDT) due to the data acquisition is done at the ground level and no destruction during the test, which is more advantageous than destructive method such as drilling and SPT test. This study was performed with Schlumberger configuration in three location by electrical resistivity sounding. The acquisition data uses Resistivity meter OYO 2119 McOHM-EL with the track length is $100 \mathrm{~m}$, while the data processing use IPI2Win to get a logarithmic graph between distance and resistivity and Origin to obtain graphic of resistivity and SPT. The result of this study indicates that electrical resistivity has a good correlation with SPT data. The electrical resistivity graph shows a linear increase along with increment of the depth, which is similar to the SPT graph. The slope difference at the first location is $2.44 \pm 1,197$, the second location is $2.028 \pm 0.822$, and the third location is $0.622 \pm 0.735$.
\end{abstract}

Keywords: resistivity, schlumberger, drilling, SPT

\section{INTRODUCTION}

Every construction investigation on the site that be built to ensure the strength of the subsurface layer ${ }^{[1]}$.Soil drilling and the Standard Penetration Test (SPT) are the preferred way for geotechnical surveys to determine subsurface lithology and to obtain the carrying capacity of the subsurface. Drilling and SPT have a good data because the test is directly into the ground to get the actual data. Although the data can obtain the actual lithology of the subsurface, for large areas need a lot of data so it costs expensive, in addition, the method is destructive. Instead, it can use electrical resistivity method from geophysics which is non-destructive, cheaper and more effective for large areas and has a good result [2].

The use of electrical resistivity method to determine the lithology of subsurface with comparison geotechnical data has been done by ${ }^{[2]}$, in that research, the acquisition of electrical resistivity data done at the location which already have drill data. The data from the electrical resistivity then combined with geotechnical data to describe the lithological and the characteristics of the subsurface layer. Research on analysis electrical resistivity with SPT also has been done by ${ }^{[3]}$, in that research the acquisition of electrical resistivity method used tomography, then resistivity data compared with SPT in a graph. The same study was conducted by ${ }^{[4]}$, comparing drill data and Standard Penetration Test (SPT) with electrical resistivity data at four measurement points. The result conducted that the electrical resistivity data has good results and similar to the drill and SPT data. 
Electrical resistivity is one of the methods in geophysics that utilizes the distribution of the Earth's electrical current in a medium that can be used to investigate subsurface layers. The electrical resistivity method can describe the subsurface profile through the distribution of the difference in resistivity value ${ }^{[5]}$.

In principle, the electrical resistivity method injects a high-voltage current of Direct Current (DC) into the ground using two current electrodes $\mathrm{C} 1$ and $\mathrm{C} 2$ that are plugged into the ground at a certain distance. The longer the distance of the current electrode, then the flow of electricity that arises deeper. Electrical currents that arise in the ground will cause electrical voltage. The results electrical voltage is measured using the potential electrode P1 and P2, where the distance of potential electrode is shorter than the current electrode. When the distance $\mathrm{C} 1$ and $\mathrm{C} 2$ are changed, the potential difference value measured by the potential electrode will change according to the type of rock or soil at the survey location [6].

Schlumberger configuration is widely used for data acquisition because it has good results for data acquisition with deep penetration. The data acquisition with Schlumberger configuration is more efficient than other configurations because in this configuration only the current electrode will be moved, while the potential electrode remains at the center of the measuring point unless the incoming current value is very small, the potential electrodes will move. Figure 1 shows electrodes array in Schlumberger Configuration ${ }^{[7]}$.

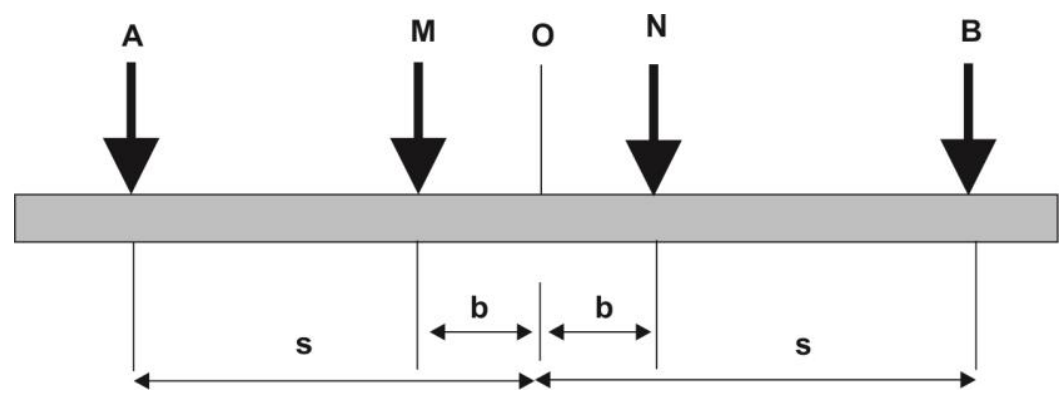

Figure 1. Electrodes array in Schlumberger Configuration ${ }^{[7]}$

In electrical resistivity method, the depiction of the earth layer is an isotropic homogeneous medium. So the measured resistivity value is considered to be a single layer resistivity value, whereas, in reality, the earth consists of layers that have different resistivity values. So the measured resistivity value of the electrical resistivity method is the apparent resistivity $(\rho)$ whose value can be determined by the following equation:

$$
\rho=K \frac{V}{I}
$$

With

$$
\begin{aligned}
& \rho=\text { Apparent resistivity }(\Omega . \mathrm{m}) \\
& K=\text { Geometry factor } \\
& V=\text { Potential }(\mathrm{mV}) \\
& I=\text { Current }(\mathrm{mA})
\end{aligned}
$$


The geometry factor value $(\mathrm{K})$ is determined based on the configuration used in the measurement. The geometry factor is determined by distance $\mathrm{AB}$ and $\mathrm{MN}$. The equation below is the geometry factor of Schlumberger configuration:

$$
K=\pi\left[\frac{\left(\frac{A B}{2}\right)^{2}-\left(\frac{M N}{2}\right)^{2}}{2\left(\frac{M N}{2}\right)}\right]
$$

Each material has different electrical characteristic, one of the characteristics is resistivity. Resistivity is the ability of a material to inhibit electric current. The greater the value of the resistivity, the more difficult the material is conducting electricity. Table 1 shows the resistivity values of some geological materials:

Table 1. Resistivity value of several materials ${ }^{[8]}$

\begin{tabular}{ccc}
\hline No & Material Type & Resistivity $(\mathbf{\Omega} . \mathbf{m})$ \\
\hline 1 & Galena & $3 \times 10^{-5}-3 \times 10^{2}$ \\
2 & Pyrite & $2,9 \times 10^{-5}-1,5$ \\
3 & Quartz & $300-10^{6}$ \\
4 & Rock salt & $30-10^{13}$ \\
5 & Granite & $300-1,3 \times 10^{6}$ \\
6 & Aluvium and sand & $10-800$ \\
7 & Clay (very dry) & $50-150$ \\
8 & Gravel (dry) & 1400 \\
9 & Gravel (saturated) & 100 \\
10 & Sand clay/clayey sand & $30-215$ \\
12 & Sand and gravel & $30-225$ \\
\hline
\end{tabular}

Geotechnical is one of the disciplines of civil engineering that focuses on studying the natural materials that exist near the surface of the earth. In general, geotechnical include the application of soil mechanics and rock mechanics to foundation designs, retaining structures and subsurface structures ${ }^{[9]}$. There are several methods that are often used for geotechnical investigation, such as drilling and Standard Penetration Test (SPT).

Drilling is one method in geotechnical that performs direct measurements into the soil. Drilling is done by making a hole in the ground using a manual drill or machine with the desired depth. At the time of drilling process, we get cutting data which is scouring from the drill bit. The cutting data is taken every $1.5 \mathrm{~m}$ depth to be used as a representation of lithology under the surface. Drilling method is usually followed by an SPT test ${ }^{[9]}$.

Standard Penetration Test (SPT) is one of the useful field tests to distinguish a layer with large obstacles with a layer of small obstacles. The large number of $\mathrm{N}$ punches will provide relative density information from sand or gravel, or about other land-type barriers to penetration. The value derived from this SPT is useful for providing information on soil 
conditions ${ }^{[10]}$. Table 2 shows SPT blows count versus relative density of sand and consistency of clay.

Table 2. SPT blow count versus relative density of sand and consistency of clay ${ }^{[11]}$

\begin{tabular}{cccc}
\hline $\begin{array}{c}\text { Penetration Resistance } \\
\text { N (blows/ft) }\end{array}$ & Density & $\begin{array}{c}\text { Penetration Resistance N } \\
\text { (blows/ft) }\end{array}$ & Consistency \\
\hline $0-4$ & Very loose & $<2$ & Very soft \\
$5-10$ & Loose & $2-4$ & Soft \\
$11-24$ & Medium & $4-8$ & Medium \\
$25-50$ & Dense & $8-15$ & Stiff \\
$>50$ & Very Dense & $15-30$ & Very stiff \\
& & $>30$ & Hard \\
\hline
\end{tabular}

\section{METHOD}

The location of the data acquisition are in three areas close to UNS campus, east Surakarta City. Based on the Surakarta-Giritontro sheet geological map, the structure of the location is alluvium where the components are clay, silt, sand, and gravel. Figure 2 shows the location of electrical resistivity data collection based on the location of the drill log and SPT data.

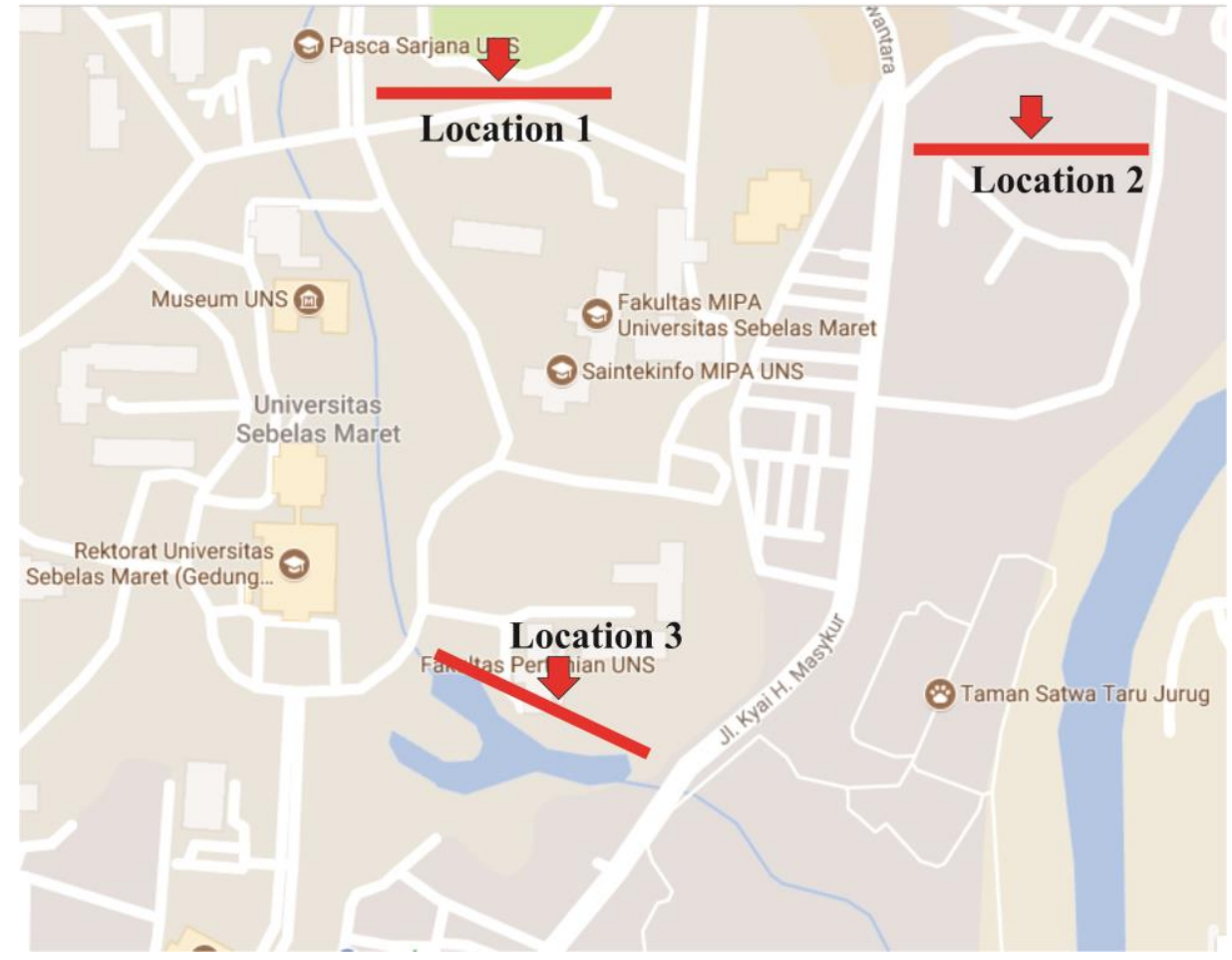

Figure 2. The acquisition data location

The equipment used for the acquisition of electrical resistivity are Resistivitymeter OYO Model 2119 McOHM-EL, electrodes, multimeter, 12Volt accumulator, cable rolls, meter roller, Global Positioning System (GPS), Handy Talky ( HT). Figure 3 shows a set of resistivitymeter for the acquisition: 


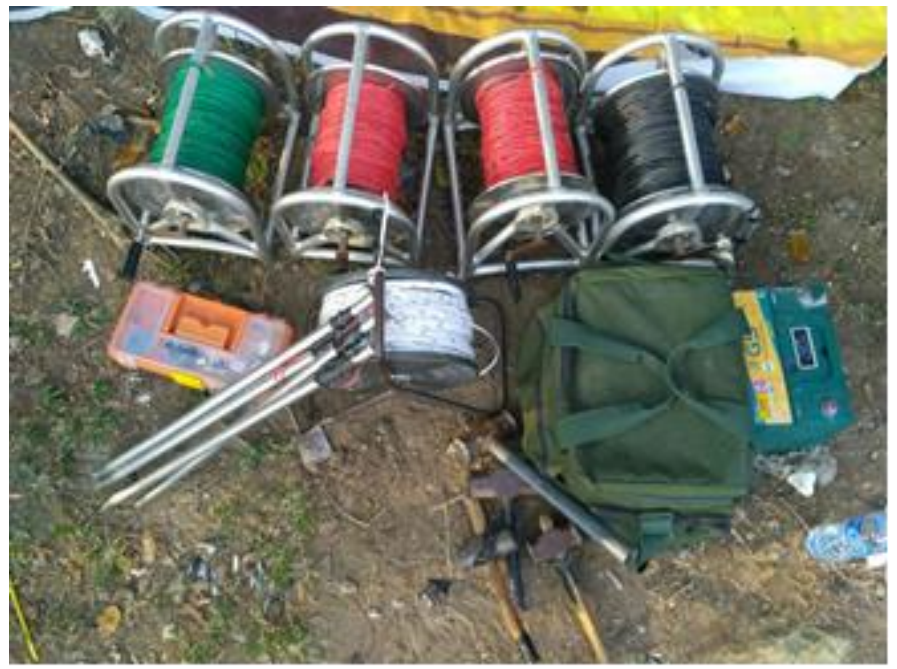

Figure 3. A set of OYO resistivitymeter

\section{RESULT AND DISCUSSION}

The resistivity data processing obtained from the acquisition is done by using IPI2Win software to obtain the logarithmic graph between the distance as the $\mathrm{x}$-axis and the apparent resistivity value as the y-axis. From the graph obtain the surface layers of depth, thickness, and the real resistivity value. The results of the data interpretation are then made a linear graphically using Origin software to see the trend line of electrical resistivity and $\mathrm{N}$-SPT data, then it will calculate the difference between the N-SPT gradient and the resistivity.

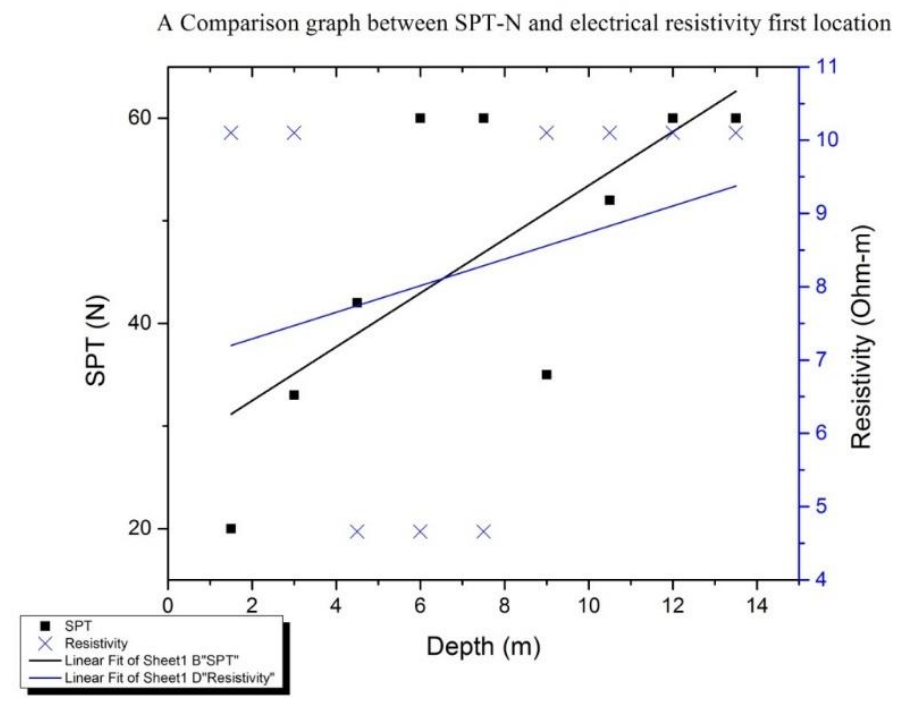

Figure 4. A Comparison graph between SPT-N and electrical resistivity first location

Figure 4 shows the SPT value increased with increasing depth, as well as the electrical resistivity results. The results of electrical resistivity at the depth of $1.5 \mathrm{~m}$ shows a high resistivity that is $10 \Omega . \mathrm{m}$ while the SPT value is low which is 20 , it means the layer at the depth of $1.5 \mathrm{~m}$ is sand with medium density. At the depth of $6 \mathrm{~m}$ and $7.5 \mathrm{~m}$, the resistivity value is low which is $4.5 \Omega$.m whereas the SPT is high which is 60 , if correlated to the depth, the layer is a silt with hard concentration. The results from the graph obtained SPT 
gradient value is $2.622 \pm 0.957$ while the resistivity gradient of Schlumberger configuration is $0.181 \pm 0.240$. The slope difference of SPT and resistivity at first location is $2,441 \pm 1,197$.

A Comparison graph between SPT-N and electrical resistivity second location

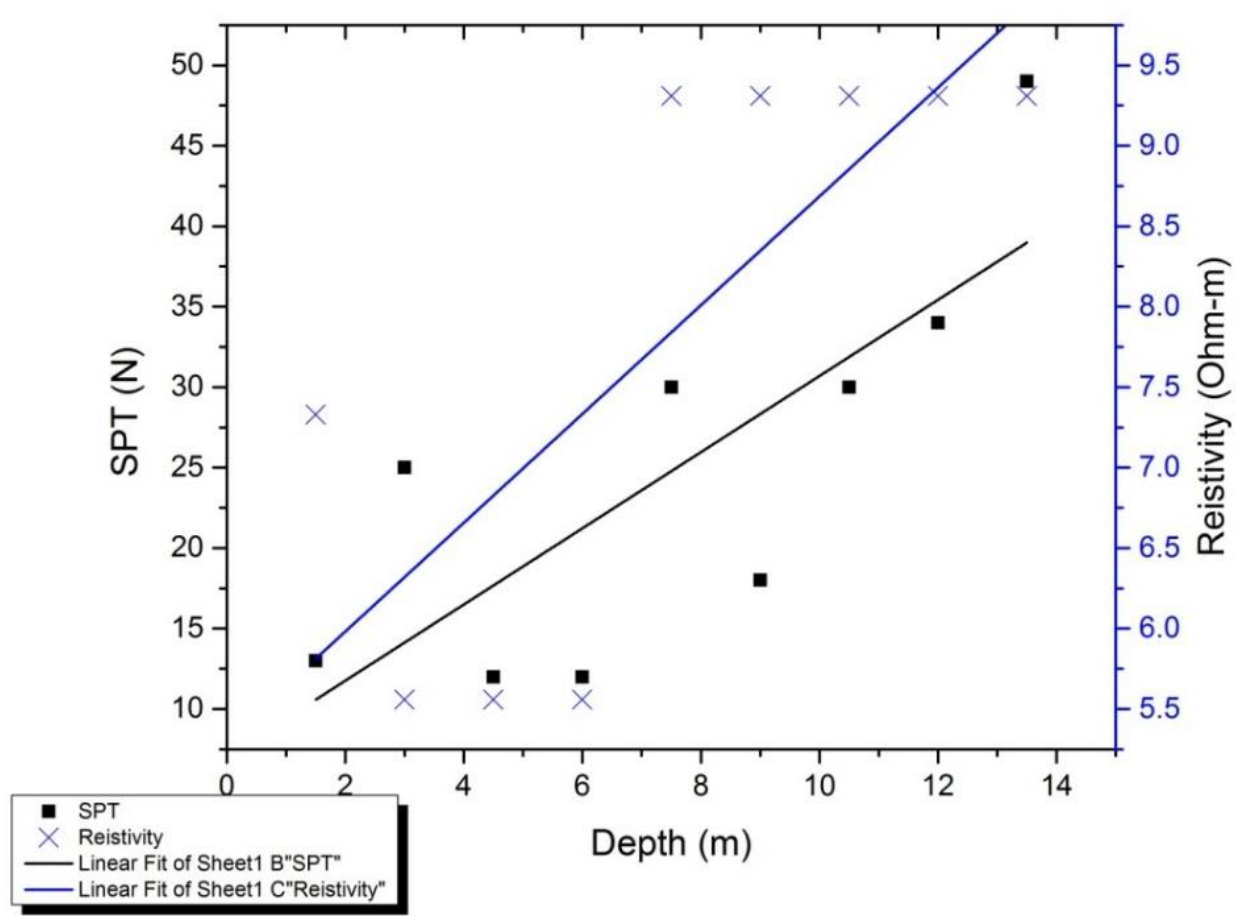

Figure 5. A Comparison graph between SPT-N and electrical resistivity second location

Figure 5 shows the SPT and electrical resistivity values increased with increasing depth. At the depth of $3 \mathrm{~m}$ the resistivity value is low which is $6 \Omega . \mathrm{m}$ whereas the value of SPT is 25 , it can be correlated at that depth, the layer is silt with very stiff consistency. At the depth of $4.5 \mathrm{~m}$ and $6 \mathrm{~m}$, both of the SPT value and resistivity have low values, it can be known the layer at that depth is a silt with a stiff consistency. At the depth of $13.5 \mathrm{~m}$ is known to be the hardest layer with SPT value is 49 and a high resistivity value which is $10 \Omega . \mathrm{m}$, based on these two data it is known the layer at this depth is dense sand. The results from the graph obtained SPT gradient value is $2.366 \pm 0.713$ while the resistivity gradient of Schlumberger configuration is $0.338 \pm 0.109$. The slope difference of SPT with resistivity at second location is $2.028 \pm 0.822$. 


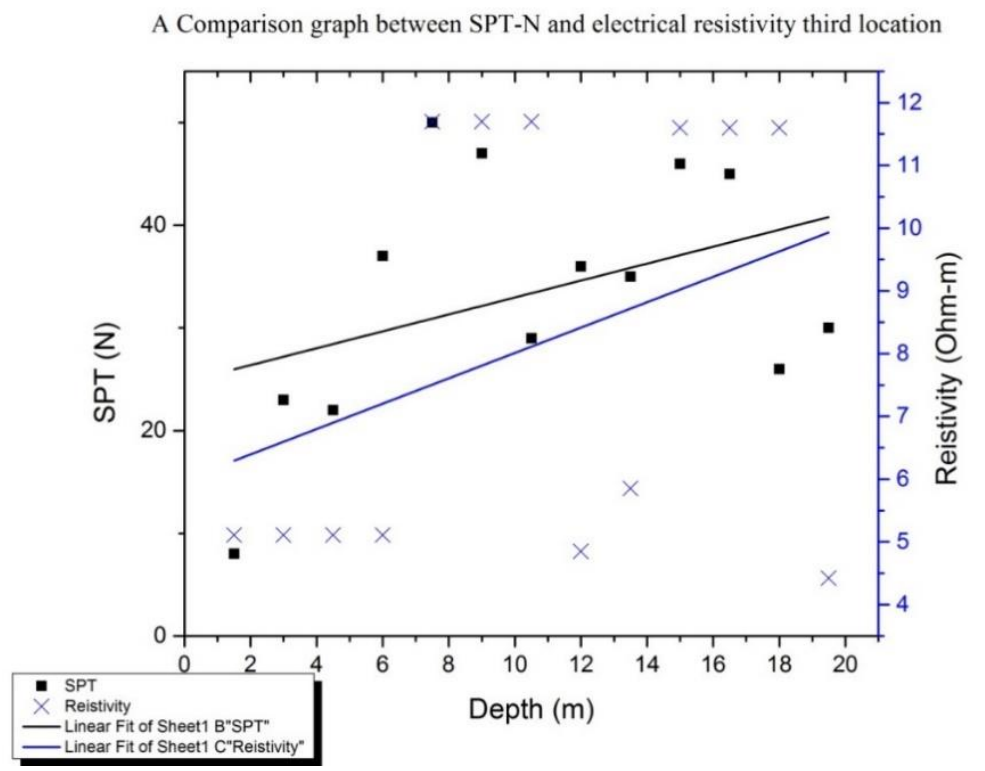

Figure 6. A Comparison graph between SPT-N and electrical resistivity third location

In Figure 6 the depth is $20 \mathrm{~m}$ and shows the SPT value increased with increasing depth, as well as the electrical resistivity results, this can be seen from the obtained trend line. At the depth of $6 \mathrm{~m}$ is known the value of SPT is 37 while the resistivity value is low which is 5 $\Omega . m$, it can be correlated that at this depth the layer is silt with a hard consistency. At the depth of $8 \mathrm{~m}$, both SPT and resistivity values are equally high, so it can be correlated the layer at this depth is sand with dense consistency. At the depth of $19.5 \mathrm{~m}$, the value of SPT shows 30 whereas the resistivity is $4.5 \Omega . \mathrm{m}$, this indicates that the layer is a silt with a very stiff consistency. Results from the graph obtained SPT gradient value is $0.824 \pm 0.570$ while the resistivity gradient value is $0.202 \pm 0.165$. The slope difference of the SPT with resistivity at third location is $0.622 \pm 0.735$.

Based on the results and analysis of the three locations, it is known that electrical resistivity with Schlumberger configuration has good correlation with N-SPT data. The results of the trend line on electrical resistivity with SPT also show the same correlation that increased with increasing depth. At the some point, there is a low resistivity value while the SPT value is high and vice versa, it is because the SPT parameter used only density and strength, while the parameter of resistivity is not only influenced by density, but also other factors such as rock/soil material, porosity, permeability, and aquifer. Each location has difference slope, the first location is $2.44 \pm 1,197$, the second location is $2.028 \pm 0.822$, and the third location is $0.622 \pm 0.735$, it is because the subsurface layers each location are different.

\section{CONCLUSION}

The results of the interpreted data showed that the electrical resistivity value has a good correlation with SPT data. Trend line obtained from SPT is increased with increasing depth, as well as the results of electrical resistivity interpretation. Each location has different gradient values, it is because the subsurface layers each location are different. For the further researcher it is desirable to conduct research on tomography basis and if possible, the acquisition of resistivity data is performed simultaneously with geotechnical drilling and SPT. 


\section{REFERENCES}

1 Oyedele, K. F. \& Okoh, C. 2011. Subsoil Investigation Using Integrated Methods at Lagos, Nigeria. Journal of Geology and Mining Research, 3(7), 169-179.

2 Dhahir, M. \& Nsaif. 2016. Geoelectrical and Geotechnical Evaluation of Subsoil at a Purposed Engineering Site, NE of Baghdad City. Diyala Journal of Engineering Sciences, 9(2), 50-61.

3 Oh, Seokhoon \& Chang Guk S. 2008. Combined analysis of electrical resistivity and geotechnical SPT blow counts for the safety assessment of fill dam. Environ Geol (54), $31-42$

4 Nimo, O. F., Ampadu, S., \& Ayeh, F. 2013. The Use Of Resistivity Measurment to Aid in Interpreting Borehole Log in Site Investigation. SAGEEP 2013.

5 Awang, H., Namawi, M. N. M., Mohamed, Z. 2008. Enhancing Geotechnical Investigation By Geoelectrical Method - A Case Study. Journal of the Souteast Asian Geotechnical Society, 185-191

6 Reynolds, M. J. 1997. An introduction to Applied and Environmental Geophysics. Reynolds Geo-Science Ltd, UK.

7 Telford, M. W., Geldart, P. L., Sheriff, E. R. 1990. Applied Geophysics Second Edition. Cambridge University Press, Cambridge

8 Lowrie, W. 2007. Fundamentals of Geophysics Second Edition. USA: Cambridge University Press.

9 Das, M. B. 2010. Principles of Geotechnical Engineering Seventh Edition. USA: Cengage Learning

10 Verhoef, W. N. P. 1994. Geologie Voor De Civiel Ingenieur. Edisi Bahasa Indonesia. Penerbit Erlangga: Jakarta

11 Terzaghi, K., \& Peck, R. B. 1967. Soil Mechanics in Engineering Practice, 2nd Edition. New York: John Wiley. 